\section{Evelina London}

T. Woodgate ${ }^{1}$, J. Handforth ${ }^{2}$, J. Newton ${ }^{2}$, F. Chappell ${ }^{3}$

${ }^{1}$ Evelina Children's Hospital, General Paediatrics, London, United Kingdom

'Evelina Children's Hospital, Paediatric Infectious Diseases, London, United Kingdom

'Evelina Children's Hospital, Pharmacy, London, United Kingdom

\section{Background}

Streptococcus pyogenes is a common bacterial organism associated with a wide spectrum of disease in children. Here we present a case of an unusually extensive retropharyngeal abscess caused by the organism.

\section{Case Presentation}

A previously healthy 13-month-old girl presented to her local hospital with five days of fever and URTI symptoms.

Inflammatory markers were raised (CRP 410 , WCC 34.6, Neutrophils 28.0) and she showed signs of shock. She was transferred to PICU at Evelina Children's Hospital where she was treated for sepsis of unknown origin.

She was intubated and ventilated whilst in PICU and investigations were performed to locate the source of her presumed infection.

An initial echocardiogram revealed a moderately large pericardial effusion. A pericardial drain removed $60 \mathrm{ml}$ of serous fluid, yet the patient remained unwell.

A repeat echocardiogram showed resolution of the effusion, and evidence of an unexpected retrosternal collection.

\section{Further Investigation Results}

Chest wall ultrasonography demonstrated a large retrosternal abscess with extension beyond the scope of the imaging modality.

Cross-sectional CT neck/thorax revealed a large $(3 \times 1.2 \mathrm{~cm})$ retropharyngeal abscess (Figure 1) extending into a large, septated, mediastinal collection (Figures 2 and 3 ). The mediastinal components measured $7 \times 2 \mathrm{~cm}$ anteriorly and $2.5 \times 2.3 \mathrm{~cm}$ posteriorly, with displacement of the oesophagus.

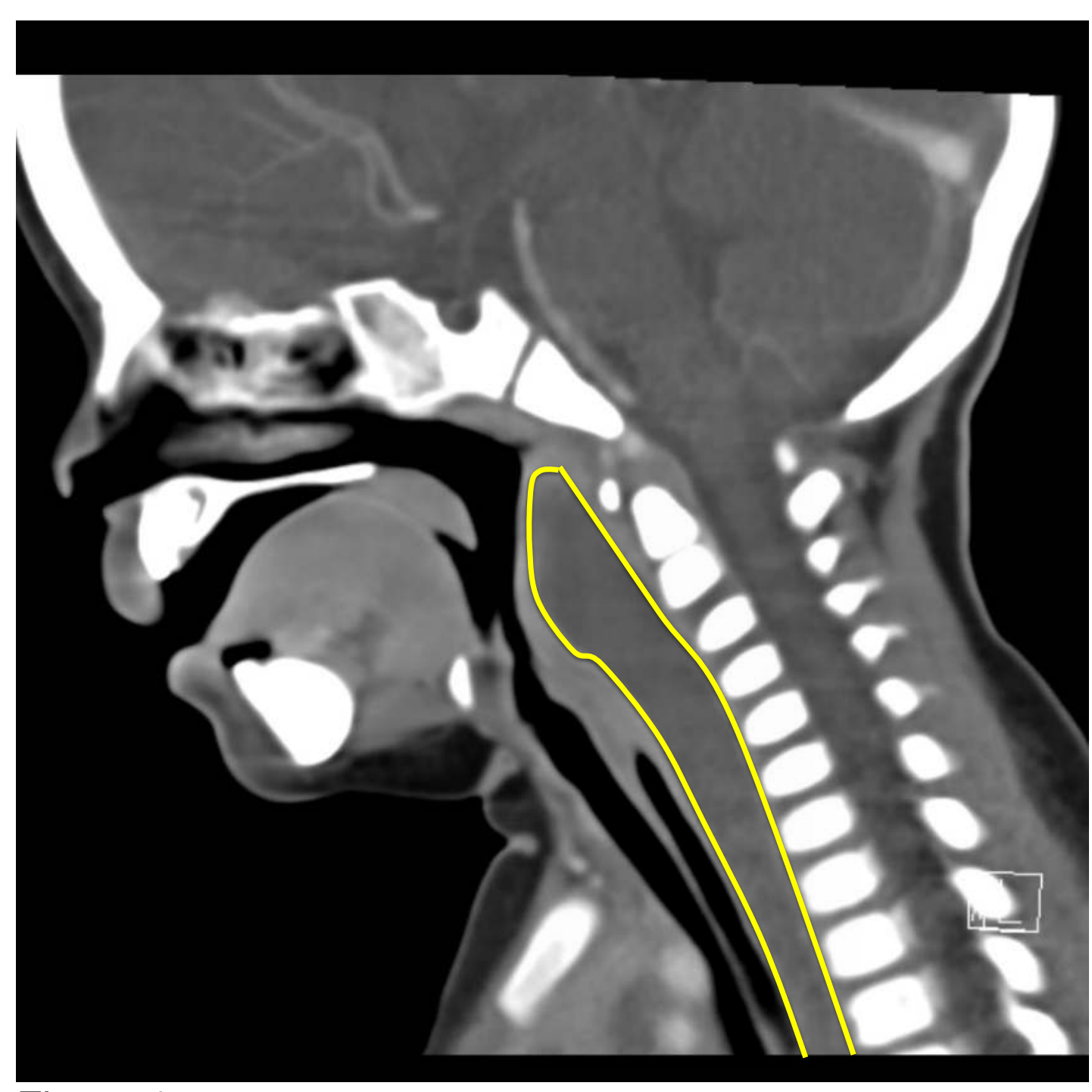

Figure 1

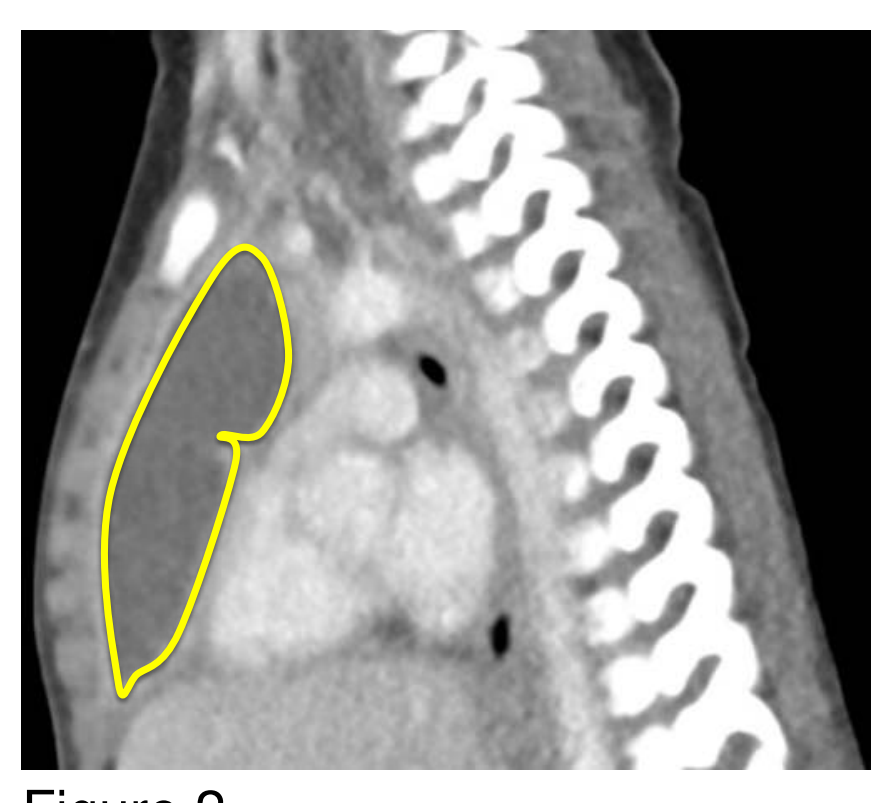

Figure 2

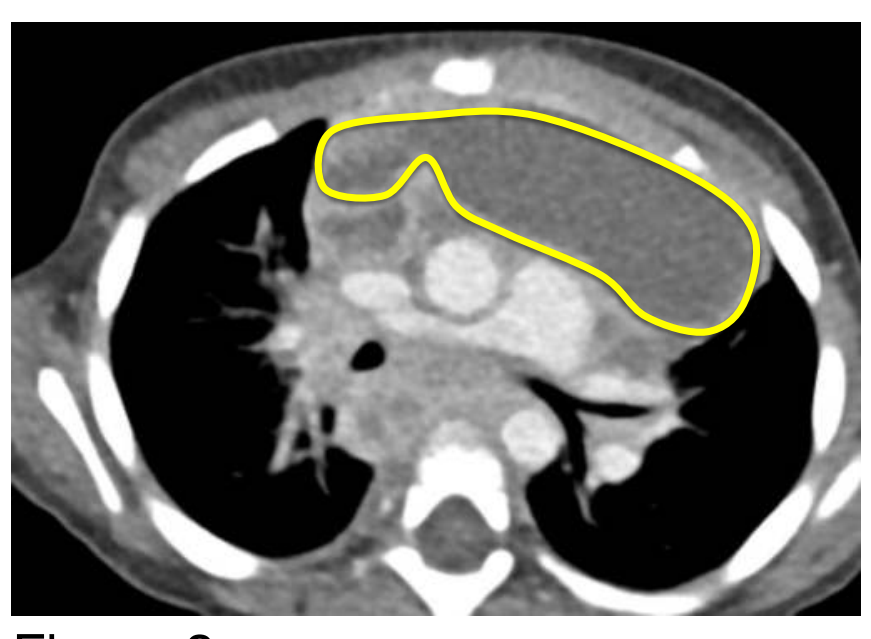

Figure 3
Oral swabs, blood cultures, and fluid cultures yielded no growth. 16S PCR analysis identified Streptococcus pyogenes in surgically drained fluid.
Treatment and Follow Up

Surgical drainage by ENT and

Cardiothoracics removed more than $25 \mathrm{ml}$ of pus, with ongoing drainage post-operatively.

Medical treatment included 8 weeks of parenteral antibiotics (ceftriaxone and clindamycin) followed by 4 weeks orally (coamoxiclav and clindamycin).

6 weeks of the parenteral therapy were delivered by $\mathrm{p}$-OPAT using a PICC.

Outpatient follow up showed that the patient made a full recovery. Immunology testing showed no evidence of immunocompromise.

\section{Learning Points}

PCR analysis was able to identify the causative organism where traditional methods had failed.

p-OPAT is an effective tool to reduce hospital stays in children with severe bacterial infections.

Streptococcus pyogenes remains a pathogen capable of causing severe disease in immunocompetent children. 